\title{
Qualidade da biomassa em híbridos de capim-elefante para fins energéticos
}

\author{
Liliane Barroso de Sousa1, Rogério Figueiredo Daher ${ }^{1}$, Bruna Rafaela da Silva Menezes², Erina Vitório Rodrigues \\ Flávio Dessaune Tardin ${ }^{3}$, Geraldo de Amaral Gravina ${ }^{1}$, Antônio Vander Pereira ${ }^{4}$ \\ 1 Universidade Estadual do Norte Fluminense Darcy Ribeiro, Centro de Ciências e Tecnologias Agropecuárias, Av. Alberto Lamego, 2000, Parque Califórnia, CEP 28015620 - \\ Campos dos Goytacazes, RJ - Brasil. E-mail: rogerio.daher@pq.cnpq.br; : lilianeuenf@hotmail.com; erinavict@hotmail.com; gravina@uenf.br \\ ${ }^{2}$ Universidade Federal Rural do Rio de Janeiro, Instituto de Ciências Biológicas e da Saúde, Departamento de Genética, Rodovia BR 465 - Km 7, Zona Rural, CEP 23897-970, \\ Seropédica-RJ, Brasil. E-mail: brunarafamenezes@gmail.com \\ ${ }^{3}$ Embrapa Agrossilvipastoril, Rodovia MT 222, Zona Rural, CEP 78550-970, Sinop-MT, Brasil. Caixa-Postal 343. E-mail: flavio.tardin@embrapa.br \\ ${ }^{4}$ Embrapa Gado de Leite, Rua Eugênio do Nascimento, 610, Dom Bosco, CEP 36038-330, Juiz de Fora-MG, Brasil E-mail: vander.pereira@embrapa.br
}

\section{RESUMO}

Além do uso como forrageira, o capim-elefante vem sendo utilizado como fonte de energia alternativa. Isso se deve pela sua alta capacidade de produção de biomassa. É desejável que essa biomassa tenha alto teor de fibras e baixo de cinzas, por isso há necessidade de se obter materiais genéticos com essas características. Com isso, o objetivo deste trabalho foi avaliar as características químicas dos híbridos de capim-elefante e dos seus genitores para produção de biomassa energética. 0 experimento foi conduzido na Empresa de Pesquisa Agropecuária do Estado do Rio de Janeiro, localizada em Campos dos Goytacazes, nos anos de 2011 e 2012. Foram efetuados dois cortes onde foram avaliadas: Porcentagem de cinzas (\%CIN); fibra de detergente neutro (\%FDN) e ácido (\%FDA); celulose (\%CEL); hemicelulose (\%HEM); e lignina (\%LIG). 0 delineamento foi em blocos ao acaso, com três repetições. Os tratamentos foram quatro genitores femininos, quatro genitores masculinos, 16 híbridos obtidos em esquema de cruzamento em dialelo parcial e uma testemunha. As médias foram comparadas pelo teste de Tukey $(\mathrm{P}<0,05)$. Em relação às características avaliadas no presente estudo, as combinações híbridas H1, H3, H5, H7, H9 e os genitores P4 e P6 possuem boas qualidades de biomassa e, com isso, apresentam potencial para uso para fins energéticos.

Palavras-chave: bioenergia, cinzas, Pennisetum purpureum

\section{Quality biomass in elephant grass hybrids for energy purposes}

\begin{abstract}
Besides the use as forage, elephant grass has been used as an alternative energy source. This is due to its high production capacity of biomass. It is desirable that this biomass has high fiber content, low ash, so there need to obtain a genetic material with these traits. Thus, the aim of this study was to evaluate the chemical characteristics of elephant grass hybrids and their parents for the production of energy biomass. The experiment was conducted at Empresa de Pesquisa Agropecuária do Estado do Rio de Janeiro, located in Campos, in the years 2011 and 2012 were made two cuts which were assessed: Percentage of ash (\% CIN); neutral detergent fiber (FDN\%) and acid (\% FDA); cellulose (CEL\%); hemicellulose (HEM\%); and lignin (\% LIG). The design was a randomized block design with three replications. The treatments were four female parents, four male parents, 16 hybrids obtained by crossing scheme partial diallel and a witness. The Means was compared by Tukey test $(P<0.05)$. As for the traits evaluated in this study, the hybrid combinations $\mathrm{H1}, \mathrm{H} 3, \mathrm{H} 5, \mathrm{H} 7, \mathrm{H} 9$ and parents $\mathrm{P} 4$ and $\mathrm{P} 6$ have good biomass qualities and, therefore, have the potential for use for energy purposes.
\end{abstract}

Key words: bioenergy, ash, Pennisetum purpureum 


\section{Introdução}

O uso da biomassa vegetal possui vantagens para a utilização como fonte alternativa de energia, pois se trata de uma fonte renovável, "energia limpa". Uma das principais espécies vegetais utilizada é o capim-elefante (Pennisetum purpureum) (Jank et al., 2013). O capim-elefante é uma forrageira com alto potencial para produção de matéria seca e de grande eficiência fotossintética (Silva et al., 2010), sendo esta uma característica típica das gramíneas tropicais que crescem rapidamente e otimizam o uso da água do solo e da energia solar (Urquiaga et al., 2012).

Para considerar a viabilidade da utilização de uma espécie vegetal para a produção de biomassa para fins energéticos é importante considerar a disponibilidade geográfica dos diferentes materiais lignocelulósicos, calendário da safra, desempenho e utilização (Cordona et al., 2012). É uma espécie perene e tem potencial para elevada produtividade, principalmente nos dois primeiros anos após a implantação do cultivo (Knoll et al., 2012).

Na cultura do capim elefante se observa grande variabilidade genética (Meinerz et al., 2011). Porém, existe a necessidade de seleção de materiais adaptados a diferentes ecossistemas existentes no Brasil. É possível a obtenção de híbridos de capim-elefante com desempenho produtivo superior a de seus genitores (Menezes et al., 2016). De acordo com Pereira (2001), a hibridação em capim-elefante constitui a melhor forma de obtenção de clones superiores, sendo que a escolha dos genitores deve ser feita com base na complementariedade alélica e na divergência genética.

O capim-elefante possui boas propriedades para uso energético, como baixo teor de cinzas, altas relações carbono/ nitrogênio no colmo e altos teores de carbono e hidrogênio (Paterlini, et al., 2013). A produção de biomassa é positivamente correlacionada com o teor de fibras e de cinzas (Rossi et al., 2014). É desejável a seleção de materiais que possuam alta produtividade e alto teor de fibras, mas com baixo teor de cinzas. Isso porque materiais com maiores teores de cinzas produzem mais voláteis a altas temperaturas, podendo inviabilizar sua utilização em para finalidade energética (Flores et al., 2013).

Sendo assim, o objetivo do trabalho foi avaliar as características químicas dos híbridos de capim-elefante e dos seus genitores para produção de biomassa energética.

\section{Material e Métodos}

O experimento foi conduzido no Centro Estadual de Pesquisas em Agroenergia e Aproveitamento de Resíduos da Empresa de Pesquisa Agropecuária do Estado do Rio de
Janeiro - PESAGRO-RJ, localizado no município de Campos

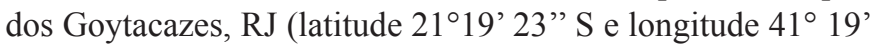
40 " W e altitude variando de 20 a $30 \mathrm{~m}$ ). O clima da região é tipo Aw de Köppen (tropical com estação seca de inverno).

Foi utilizado o delineamento de blocos casualizados com três repetições. A parcela foi composta por uma linha de $4 \mathrm{~m}$ com espaçamento de 1,5 m entre linhas, sendo considerada útil $1 \mathrm{~m}$ dentro das linhas. Cada bloco foi composto por 25 tratamentos (16 híbridos, 8 genitores e 1 testemunha).

O plantio foi realizado em maio de 2010, por meio de estacas dispostas pé com ponta, distribuídas em sulcos de 10 $\mathrm{cm}$ de profundidade. No plantio foram incorporados $100 \mathrm{~kg}$ $\mathrm{ha}^{-1}$ de $\mathrm{P}_{2} \mathrm{O}_{5}$ (superfosfato simples). A irrigação foi oferecida apenas durante a emergência das plantas e, após 50 dias de plantio, complementou-se a adubação com $25 \mathrm{~kg} \mathrm{ha}^{-1}$ de sulfato de amônio e cloreto de potássio.

Após a fase de estabelecimento, todos os genótipos foram cortados rente ao solo (corte de uniformização) no dia 17 de dezembro de 2010, em seguida foi feita uma adubação em cobertura com $25 \mathrm{~kg} \mathrm{ha}^{-1}$ de sulfato de amônio e cloreto de potássio. Nas parcelas em que houve falhas na emergência das plantas, procedeu-se ao replantio.

O $1^{\circ}$ corte foi feito em 25 de agosto de 2011 (36 semanas após o corte de uniformização - período seco) e o $2^{\circ}$ corte, em 16 de abril de 2012 (36 semanas após o $1^{\circ}$ corte - período chuvoso).

Foram utilizados dois grupos de genitores de capim-elefante. O grupo 1 constituído por quatro genitores masculinos: Taiwan A-144 (P1), Vruckwona (P2), Pusa Napier n ${ }^{\circ} 2$ (P3) e Porto Rico 534-B (P4). E o grupo 2, formado por quatro genitores femininos: Mercker Santa Rita (P5), Taiwan A-146 (P6), Mercker S. E. A. (P7) e Napier $n^{\circ} 2$ (P8). Como testemunha, foi utilizado o acesso BAG-86. As 16 combinações híbridas obtidas dos cruzamentos dialélicos estão apresentados na Tabela 1 .

As características de qualidade de biomassa avaliadas foram: porcentagem de cinza $(\% \mathrm{CIN})$, estimada pela da razão entre o peso da amostra seca na mufla $600{ }^{\circ} \mathrm{C}$ por $15 \mathrm{~h}$ e o peso da matéria seca definitiva (Silva \& Queiroz, 2002); porcentagem de hemicelulose (\%HEM): estimada pela diferença entre as porcentagens obtidas de $\%$ FDN (porcentagem de fibras em detergente neutro) e \%FDA (porcentagem de fibras em detergente ácido); e as análises das \%FDN, \%FDA, \%CEL (porcentagem de celulose) e \%LIG (porcentagem de lignina) foram feitas pelo Laboratório de Análise de Alimentos da Embrapa Gado de Leite e efetuadas por espectrofotometria próxima do infravermelho (NIRS). Todas as análises foram feitas com base na planta inteira.

Inicialmente, foi realizada uma análise de variância individual pelo teste F para cada corte. Depois de verificada a

Tabela 1. Esquema dos cruzamentos do dialelo parcial com oito genitores e dezesseis combinações híbridas de capim-elefante

\begin{tabular}{|c|c|c|c|c|}
\hline \multirow{2}{*}{$\begin{array}{c}\text { Grupo } 2 \\
\text { (genitores femininos) }\end{array}$} & \multicolumn{4}{|c|}{ Grupo 1 (genitores masculinos) } \\
\hline & $\begin{array}{c}\text { Taiwan A -144 } \\
\text { (P1) }\end{array}$ & $\begin{array}{c}\text { Vruckwona } \\
\text { (P2) }\end{array}$ & $\begin{array}{c}\text { Pusa Napier } n^{0} 2 \\
\text { (P3) }\end{array}$ & $\begin{array}{c}\text { Porto Rico 534-B } \\
\text { (P4) }\end{array}$ \\
\hline Mercker Santa Rita (P5) & $\mathrm{H} 1$ & $\mathrm{H} 5$ & $\mathrm{H} 9$ & $\mathrm{H} 13$ \\
\hline Taiwan A -146 (P6) & $\mathrm{H} 2$ & $\mathrm{H} 6$ & $\mathrm{H} 10$ & $\mathrm{H} 14$ \\
\hline Mercker S. E. A. (P7) & $\mathrm{H} 3$ & $\mathrm{H} 7$ & $\mathrm{H} 11$ & $\mathrm{H} 15$ \\
\hline Napier $n^{0} 2$ (P8) & $\mathrm{H} 4$ & $\mathrm{H} 8$ & $\mathrm{H} 12$ & $\mathrm{H} 16$ \\
\hline
\end{tabular}


homogeneidade das variâncias residuais, foi realizada a análise de variância conjunta dos dois cortes de avaliação, segundo o delineamento de parcelas subdivididas no tempo (Steel \& Torrie, 1980). Após a análise de variância de cada corte, as médias dos tratamentos foram comparadas pelo teste de Tukey a $5 \%$ de probabilidade. A análise estatística foi feita utilizando o programa GENES (Cruz, 2013).

\section{Resultados e Discussão}

Evidenciou-se diferença significativa ao nível de $1 \%$ de probabilidade (Tabela 2), pelo teste F, para a característica $\% \mathrm{FDN}$, nas fontes de variação tratamentos e genótipos, e para a característica \%CEL, na fonte de variação genitores. Foram significativas ao nível de $5 \%$ as características $\% \mathrm{CIN}$ e $\% \mathrm{FDA}$, nas fontes de variação tratamentos, genótipos, genitores e o contraste Híbridos vs genitores ( $\mathrm{H}$ vs g); \%FDN, nas fontes de variação híbridos e genitores; \%CEL, nas fontes tratamentos, genótipos e no contraste $\mathrm{H}$ vs $\mathrm{g}$; e \%HEM, nas fontes tratamentos, genótipos e híbridos. A característica \%LIG foi não significativa em todas as fontes de variação.

Com relação aos coeficientes de variação experimental (CVe), as características \%FDN, \%HEM, \%CEL, e \%FDA apresentaram valores reduzidos de coeficiente de variação experimental, 2,13;2,56;2,72 e 3,14\%, respectivamente.
Embora a característica \%LIG não tenha sido significativa, o valor do $(\mathrm{CVe}=6,84 \%)$ foi menor que o $\mathrm{CVe}$ da $\% \mathrm{CIN}$, cujo valor foi igual a $14,09 \%$. Desta forma, verifica-se que a maioria das características de qualidade de biomassa avaliada apresentou coeficiente de variação considerado baixos (Pimentel-Gomes, 2009). Esse resultado indica que há maior participação da variação genética na variação fenotípica (Assis et al., 2010). Essa maior participação da variação genética na variação fenotípica é favorável durante o processo de seleção dos melhores genótipos, pois se estará selecionando com base na proporção herdável da variância fenotípica.

No $2^{\circ}$ corte (Tabela 3 ), ocorreram diferenças em relação ao $1^{\circ}$ corte como no caso das características que antes foram significativas e no $2^{\circ}$ corte foram não significativas como para a \%CIN e \%HEM e também para \%LIG, que antes não havia sido significativa e neste corte foi significativa a $5 \%$ nas fontes de variação tratamentos, genótipos e híbridos. Além disso, foi observada diferença significativa a $1 \%$ de probabilidade para as características \%FDN, \%FDA e \%CEL, nas fontes de variação tratamentos, genótipos e híbridos. As características $\%$ FDN e \%CEL também foram significativas na fonte de variação genitores, ou seja, a média dos genitores avaliados para as características \%FDN e \%CEL diferiram entre si.

Quanto aos valores dos coeficientes de variação experimental $(\mathrm{CVe})$, também ocorreram alterações, tendo

Tabela 2. Resumo da análise de variância das seis características da qualidade da biomassa avaliadas no $1^{\circ}$ corte, envolvendo os 16 híbridos resultantes dos cruzamentos dialélicos, os oito genitores de capim-elefante (Pennisetum purpureum Schum.) e a testemunha (BAG-86)

\begin{tabular}{|c|c|c|c|c|c|c|}
\hline FV & $\% \mathrm{CIN}$ & $\%$ FDN & $\%$ FDA & $\%$ CEL & \%HEM & $\%$ LIG \\
\hline Blocos & 3,446 & 8,746 & 1,448 & 0,294 & 5,106 & 1,245 \\
\hline Tratamentos & $0,709^{*}$ & $6,368^{* *}$ & $4,80^{*}$ & $2,616^{*}$ & $1,052^{*}$ & $0,553^{\text {ns }}$ \\
\hline Genótipos (G) & $16,777^{*}$ & $6,464^{* *}$ & $4,629^{*}$ & $2,611^{*}$ & $1,070^{*}$ & $0,552^{\mathrm{ns}}$ \\
\hline Híbridos $(\mathrm{H})$ & $0,595^{\mathrm{ns}}$ & $5,388^{*}$ & $2,941^{\mathrm{ns}}$ & $1,215^{\mathrm{ns}}$ & $1,202^{*}$ & $0,463^{\text {ns }}$ \\
\hline Genitores (g) & $0,901^{*}$ & $8,282^{*}$ & $7,178^{*}$ & $4,786^{* *}$ & $0,858^{\mathrm{ns}}$ & $0,691^{n s}$ \\
\hline G vs testemunha & $0,255 \mathrm{~ns}$ & $8,282^{*}$ & $8,729^{n s}$ & $2,742^{\text {ns }}$ & 0,639 ns & $0,591^{\mathrm{ns}}$ \\
\hline $\mathrm{H}$ vs g & $1,538^{*}$ & $9,880^{\text {ns }}$ & $12,104^{*}$ & $8,308^{*}$ & $0,567^{\mathrm{ns}}$ & $0,908^{\text {ns }}$ \\
\hline Resíduos & 0,376 & 2,780 & 2,463 & 1,202 & 0,533 & 0,358 \\
\hline Média geral & 4,35 & 78,44 & 49,99 & 40,25 & 28,51 & 8,76 \\
\hline Média dos genótipos & 4,36 & 78,49 & 50,06 & 40,29 & 28,49 & 8,77 \\
\hline Média dos híbridos & 4,47 & 78,23 & 49,78 & 40,06 & 28,56 & 8,70 \\
\hline Média dos genitores & 4,16 & 79,01 & 50,65 & 40,78 & 28,37 & 8,94 \\
\hline Média da testemunha & 4,07 & 77,29 & 48,32 & 39,32 & 28,96 & 8,33 \\
\hline CVe $(\%)$ & 14,09 & 2,13 & 3,14 & 2,72 & 2,56 & 6,84 \\
\hline
\end{tabular}

\%CIN: porcentagem de cinza; \%FDN: porcentagem de fibra em detergente neutro; \%FDA: porcentagem de fibra em detergente ácido; \%CEL: porcentagem de celulose; \%HEM: porcentagem de hemicelulose; \%LIG: porcentagem de lignina; CV e: coeficiente de variação experimental; ** significativo a $1 \%$ de probabilidade; * ${ }^{2}$ significativo a $5 \%$ de probabilidade; ns: não significativo pelo Teste F.

Tabela 3. Resumo da análise de variância das seis características da qualidade da biomassa avaliadas no $2^{\circ}$ corte, envolvendo os 16 híbridos resultantes dos cruzamentos dialélicos, os oito genitores de capim-elefante (Pennisetum purpureum Schum.) e a testemunha (BAG-86)

\begin{tabular}{|c|c|c|c|c|c|c|}
\hline FV & $\% \mathrm{CIN}$ & $\%$ FDN & \%FDA & $\%$ CEL & \%НЕМ & $\%$ LIG \\
\hline Blocos & 0,010 & 7,808 & 12,365 & 7,569 & 0,304 & 0,086 \\
\hline Tratamentos & $1,145^{\text {ns }}$ & $5,940^{* *}$ & $7,474^{* *}$ & $4,132^{* *}$ & $0,914^{\mathrm{ns}}$ & $1,457^{\star}$ \\
\hline Genótipos (G) & $1,162^{\text {ns }}$ & $6,104^{* *}$ & $7,747^{* *}$ & $4,171^{* *}$ & $0,945^{\text {ns }}$ & $1,516^{*}$ \\
\hline Híbridos $(\mathrm{H})$ & $0,918^{\text {ns }}$ & $6,246^{* *}$ & $7,645^{\star *}$ & $3,760^{* *}$ & $0,993^{\text {ns }}$ & $1,587^{\star}$ \\
\hline Genitores (g) & $1,627^{\mathrm{ns}}$ & $5,510^{* *}$ & $8,214^{*}$ & $4,559^{* *}$ & $0,974^{\text {ns }}$ & $1,581^{\mathrm{ns}}$ \\
\hline G vs testemunha & $0,760^{\text {ns }}$ & 2,159 ns & 1,211 ns & $3,232^{\text {ns }}$ & $0,206^{\text {ns }}$ & $0,100^{\text {ns }}$ \\
\hline $\mathrm{H}$ vs g & $1,565^{\text {ns }}$ & $8,192^{*}$ & $5,990^{\text {ns }}$ & $7,670^{*}$ & $0,122^{\text {ns }}$ & $0,135^{\text {ns }}$ \\
\hline Resíduos & 0,760 & 1,713 & 2,945 & 1,265 & 0,606 & 0,726 \\
\hline Média geral & 4,83 & 81,06 & 53,84 & 45,15 & 27,26 & 7,84 \\
\hline Média dos genótipos & 4,85 & 81,09 & 53,87 & 45,19 & 27,27 & 7,83 \\
\hline Média dos híbridos & 4,96 & 80,86 & 53,67 & 44,97 & 27,26 & 7,84 \\
\hline Média dos genitores & 4,65 & 81,57 & 54,28 & 45,66 & 27,29 & 7,85 \\
\hline Média da testemunha & 4,34 & 80,23 & 53,22 & 44,13 & 27,00 & 8,02 \\
\hline CV e (\%) & 18,04 & 1,61 & 3,19 & 2,49 & 2,86 & 10,86 \\
\hline
\end{tabular}

\%CIN: porcentagem de cinza; \%FDN: porcentagem de fibra em detergente neutro; \%FDA: porcentagem de fibra em detergente ácido; \%CEL: porcentagem de celulose; \%HEM: porcentagem de

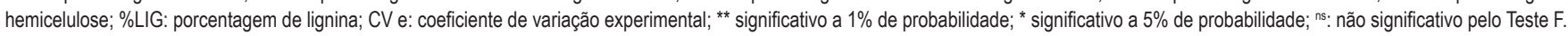


sido observada uma ordem crescente de valores, de modo que a $\%$ FDN apresentou o menor valor $(1,61 \%)$ seguida das características \%CEL $(\mathrm{CVe}=2,49 \%), \% \mathrm{HEM}(\mathrm{CVe}=2,86 \%)$, $\%$ FDA $(\mathrm{CVe}=3,19 \%)$. As características \%LIG e \%CIN apresentaram mais uma vez os valores mais altos de CVe 10,86 e $18,04 \%$, respectivamente. Esses valores mais elevados para indicam influência ambiental mais elevada nas características \%LIG e \%CIN em comparação as demais características de qualidade de biomassa avaliadas no presente estudo.

Houve homocedasticidade da variância do erro, com base na relação entre o maior quadrado médio do resíduo (QMR) e o menor quadrado médio do resíduo (QMR): para a \%CIN, o resultado foi 2,034; para a $\% \mathrm{FDN}$, o valor foi de 1,444; para $\%$ FDA, de 1,219; para \%CEL, de 1,189; para \%HEM, de 1,141 ; e para $\%$ LIG, de 2,350. Estes resultados atenderam a exigência de serem menor ou igual a sete, possibilitando que os dados do $1^{\circ}$ e do $2^{\circ}$ corte fossem analisados de forma conjunta.

De acordo com os resultados da Tabela 4, ocorreram diferenças significativas, pelo teste $\mathrm{F}$ entre os genótipos em quase todas as características avaliadas, com exceção para $\%$ HEM, o que indica a existência de variabilidade genética entre os tratamentos. Resultado semelhante foi encontrado por Oliveira et al. (2015) onde a característica \%HEM não apresentou diferenças significativas a 5\% de probabilidade. Os autores atribuíram esse fato ao coeficiente de variação genético ser mais elevado em relação ao coeficiente de variação experimental nessa característica. Além disso, observouse que entre os cortes ocorreram diferenças significativas a $1 \%$ na $\%$ CEL e a $5 \%$ de probabilidade nas características $\%$ FDN, \%FDA, \%HEM e \%LIG e ausência de significância na \%CIN. Na interação genótipos x cortes $(\mathrm{G} \times \mathrm{C})$, ocorreram diferenças na característica \%FDN a $1 \%$ de probabilidade e nas características \%FDA, \%CEL, \%HEM, \%LIG a 5\% de probabilidade. Estes resultados indicam que alguns genótipos sofreram influência do corte, ou seja, o desempenho desses genótipos para as características avaliadas pode variar de acordo com o corte de avaliação, que está relacionada com as condições ambientais (temperatura, luminosidade, distribuição da precipitação pluvial) predominantes durante o período de crescimento da cultura.

$\mathrm{Na}$ característica \%CIN, novamente foi constatada ausência de significância. Neste tipo de análise, existem três tipos de erros, cada erro relacionado a uma fonte da variação, ou seja, o CV (\%) do erro a está relacionado aos genótipos, o do erro b está ligado aos cortes e do erro $\mathrm{c}$ está vinculado à interação (G x C). Neste estudo, foram considerados apenas os coeficientes de variação do erro a cujos valores variaram entre $17,07 \%$ na $\% \mathrm{CIN}$ a $2,12 \%$ na $\% \mathrm{FDN}$ (Tabela 4). Leão et al. (2012), avaliando híbridos entre genótipos de capim-elefante e milheto, encontraram valores de coeficiente de variação de $1,26 \%$ para a característica $\% \mathrm{FDN}$, ou seja, valor mais baixo do que o encontrado no presente experimento.

Na Tabela 5, após analisar as médias verticalmente, observou-se que no $1^{\circ}$ corte a média variou entre $5,42 \%$, no H12, a 3,26\%, no genitor P4. Além disso, foram detectadas diferenças entre os genótipos H12, H13, cujas médias foram $5,42 \%, 5,39 \%$, respectivamente. O genitor Porto Rico 534-B apresentou média de $3,26 \%$. No $2^{\circ}$ corte, o valor médio de cada genótipo foi superior ao do $1^{\circ}$ corte, com exceção dos híbridos $\mathrm{H} 4, \mathrm{H} 8, \mathrm{H} 13$ e dos genitores P5 e P8, que apresentaram redução dos seus respectivos valores médios. De acordo com Moraes et al. (2009) para o capim-elefante o teor de cinzas deve ser ao redor de $5 \%$. Acima disso, o uso desse material para fins energético estaria comprometido. $O$ valor médio para $\%$ CIN foi encontrado para a combinação híbrida de 5,76\% no $2^{\circ}$ corte, ou seja, os materiais aqui avaliados apresentaram níveis satisfatórios de \%CIN.

$\mathrm{Na}$ característica \%FDN, reconhecidamente de grande importância na avaliação para a produção de biomassa energética, pois é desejável materiais com altos teores de fibras, a média observada foi de $79,75 \%$ (Tabela 5). No $1^{\circ}$ corte, constatou-se que o híbrido H16, com média 74,55\%, e o P2, com média de $75,85 \%$, diferiram do genitor Pusa Napier $\mathrm{n}^{\mathrm{0}} 2$, com média de $81,67 \%$, sendo esta a maior média observada. No $2^{\circ}$ corte, a média dos genótipos foi superior à do $1^{\circ}$ corte, variando de 84,63 a $78,68 \%$. O híbrido H16, com média de $84,63 \%$, diferiu estatisticamente dos genótipos H2, H5, H7, H9, H10, H12, H14, P2, P6 e a testemunha BAG86 , cujas médias variaram entre 80,48 e $78,68 \%$. Ao analisar o comportamento dos genótipos nos dois cortes, verificouse que entre os 25 tratamentos, os híbridos H2, H3, H4, H5, H10, H11, H12, H14 e os genitores Pusa Napier n ${ }^{\circ}$ 2, Taiwan A-146, Mercker S. E. A. não sofreram influência do corte o que é importante, pois esses genótipos não apresentariam comportamento diferente ao longo dos cortes. Isso é importante no melhoramento de plantas, pois ao selecionar esses genótipos

Tabela 4. Resumo da análise de variância conjunta das seis características da qualidade da biomassa com base em dois cortes de avaliação, envolvendo os 16 híbridos resultantes dos cruzamentos dialélicos, oito genitores de capim-elefante (Pennisetum purpureum Schum.) e a testemunha (BAG-86)

\begin{tabular}{|c|c|c|c|c|c|c|}
\hline & $\% \mathrm{CIN}$ & $\%$ FDN & $\%$ FDA & $\%$ CEL & \%НЕМ & $\%$ LIG \\
\hline Blocos & 1,563 & 0,542 & 3,092 & 2,660 & 3,369 & 0,983 \\
\hline Genótipos (G) & $1,149^{*}$ & $6,816^{* *}$ & $8,505^{* *}$ & $5,119^{* *}$ & $1,013^{\mathrm{ns}}$ & $1,147^{*}$ \\
\hline Erro a & 0,612 & 2,862 & 3,420 & 1,570 & 0,661 & 0,592 \\
\hline Cortes (C) & $8,604^{\text {ns }}$ & $257,205^{*}$ & $556,229^{*}$ & $899,248^{* *}$ & $58,631^{*}$ & $31,355^{*}$ \\
\hline Erro b & 1,893 & 16,012 & 10,722 & 5,204 & 2,041 & 0,348 \\
\hline Interação (GxC) & $0,706^{\text {ns }}$ & $5,492^{* *}$ & $3,770^{*}$ & $1,630^{*}$ & $0,953^{*}$ & $0,864^{*}$ \\
\hline Erro C & 0,521 & 1,632 & 1,990 & 0,898 & 0,4791 & 0,493 \\
\hline Média & 4,59 & 79,75 & 51,92 & 42,70 & 27,89 & 8,30 \\
\hline $\mathrm{CV}(\%)$ erro a & 17,08 & 2,12 & 3,56 & 2,93 & 2,91 & 9,26 \\
\hline $\mathrm{CV}(\%)$ erro b & 29,94 & 5,01 & 6,31 & 5,342 & 5,12 & 7,11 \\
\hline $\mathrm{CV}(\%)$ erro C & 15,71 & 1,60 & 2,72 & 2,22 & 2,48 & 8,46 \\
\hline
\end{tabular}

\% CIN: porcentagem de cinza; \%FDN: porcentagem de fibra em detergente neutro; \%FDA: porcentagem de fibra em detergente ácido; \%CEL: porcentagem de celulose; \%HEM: porcentagem de hemicelulose; \%LIG: porcentagem de lignina; ** significativo a $1 \%$ de probabilidade; * significativo a $5 \%$ de probabilidade; ns: não significativo pelo Teste $\mathrm{F}$. 
Tabela 5. Valores médios das três características da qualidade da biomassa dos 16 híbridos, dos oito genitores de capim-elefante e da testemunha (BAG-86)

\begin{tabular}{|c|c|c|c|c|c|c|}
\hline & \multicolumn{2}{|c|}{$\% \mathrm{CIN}$} & \multicolumn{2}{|c|}{$\%$ FDN } & \multicolumn{2}{|c|}{ \%FDA } \\
\hline & $1^{0}$ & $2^{\circ}$ & $1^{\mathrm{o}}$ & $2^{\circ}$ & $1^{\mathbf{o}}$ & $2^{0}$ \\
\hline$\overline{\mathrm{H} 1}$ & $4,11 \mathrm{ab} A$ & 4,65 a A & $77,97 \mathrm{abc} B$ & $81,7 \mathrm{abc} A$ & $49,63 \mathrm{ab} B$ & $54,82 \mathrm{ab} A$ \\
\hline $\mathrm{H} 2$ & $4,66 \mathrm{ab} A$ & 5,01 a $A$ & $79,13 a b c A$ & $80,05 b c A$ & $50,38 \mathrm{ab} B$ & $52,85 \mathrm{ab} A$ \\
\hline H3 & $4,31 \mathrm{ab} \mathrm{A}$ & 5,14 a $A$ & 79,63 abc A & 81,56 abc $A$ & $51,78 \mathrm{ab} A$ & $53,91 \mathrm{ab} A$ \\
\hline $\mathrm{H} 4$ & $4,87 \mathrm{ab} A$ & 4,83 a $A$ & 79,52 abc $A$ & 80,77 abc A & $50,93 \mathrm{ab} B$ & $54,33 \mathrm{ab} A$ \\
\hline H5 & $4,44 a b \mathrm{~A}$ & 5,30 a A & $78,54 a b c A$ & $80,48 \mathrm{bcA}$ & $49,48 \mathrm{ab} B$ & $53,44 \mathrm{ab} A$ \\
\hline H6 & $3,86 \mathrm{ab} \mathrm{A}$ & 4,25 a $A$ & $77,62 \mathrm{abc} B$ & $81,71 a b c A$ & $48,78 \mathrm{ab} B$ & $56,02 \mathrm{ab} \mathrm{A}$ \\
\hline H7 & $3,96 \mathrm{ab} B$ & 5,74 a A & $76,47 \mathrm{abc} B$ & 79,72 bc A & $48,58 \mathrm{ab} B$ & $52,53 \mathrm{ab} A$ \\
\hline $\mathrm{H} 8$ & $4,60 a b A$ & 3,87 a A & 79,37 abc B & $82,77 a b c A$ & $50,78 \mathrm{ab} B$ & $56,21 \mathrm{ab} A$ \\
\hline $\mathrm{H} 9$ & $4,20 \mathrm{ab} A$ & 4,85 a $A$ & $77,62 a b c B$ & $79,82 \mathrm{bcA}$ & $49,23 \mathrm{ab} B$ & $51,61 \mathrm{bA}$ \\
\hline $\mathrm{H} 10$ & $4,23 a b \mathrm{~A}$ & 5,32 a $A$ & 78,93 abc $A$ & $78,68 \mathrm{cA}$ & $50,67 \mathrm{ab} A$ & $51,25 \mathrm{~b} \mathrm{~A}$ \\
\hline $\mathrm{H} 11$ & $4,38 \mathrm{ab} \mathrm{A}$ & 5,40 a A & $79,80 \mathrm{abc} A$ & 80,81 abc $A$ & $50,07 a b B$ & $53,03 a b A$ \\
\hline $\mathrm{H} 12$ & 5,42 a $A$ & 5,76 a $A$ & $78,40 a b c A$ & $79,67 \mathrm{bcA}$ & $49,52 \mathrm{ab} B$ & $52,54 \mathrm{ab} A$ \\
\hline $\mathrm{H} 13$ & $5,39 a \mathrm{~A}$ & 4,13 a $B$ & $78,10 \mathrm{abc} B$ & $81,21 a b c A$ & $49,82 \mathrm{ab} B$ & $54,64 \mathrm{ab} A$ \\
\hline $\mathrm{H} 14$ & $4,15 a b B$ & 5,48 a A & $77,47 \mathrm{abc} A$ & $79,50 \mathrm{bcA}$ & $47,87 \mathrm{ab} B$ & $51,84 \mathrm{bA}$ \\
\hline $\mathrm{H} 15$ & $4,47 \mathrm{ab} A$ & 4,90 a A & $78,47 a b c B$ & $80,60 a b c A$ & $49,83 \mathrm{ab} B$ & $53,50 \mathrm{ab} A$ \\
\hline $\mathrm{H} 16$ & $4,43 a b \mathrm{~A}$ & 4,66 a $A$ & 74,55 c B & 84,63 a A & $49,01 \mathrm{ab} B$ & $56,18 \mathrm{ab} A$ \\
\hline $\mathrm{P} 1$ & $4,41 \mathrm{ab} \mathrm{A}$ & 4,95 a $A$ & 79,16 abc B & $83,56 \mathrm{ab} A$ & $51,07 \mathrm{ab} B$ & 57,33 a $A$ \\
\hline P2 & $4,08 a b B$ & 5,55 a A & 75,85 bc B & $79,38 \mathrm{cA}$ & 47,38 b B & $51,61 \mathrm{bA}$ \\
\hline P3 & $3,56 \mathrm{ab} A$ & 3,85 a $A$ & 81,67 a A & $82,70 a b c A$ & 52,58 a B & $55,01 \mathrm{ab} \mathrm{A}$ \\
\hline P4 & $3,26 \mathrm{bA}$ & 3,75 a A & $78,17 \mathrm{abc} B$ & $80,98 a b c A$ & $50,47 \mathrm{ab} B$ & $54,11 \mathrm{ab} A$ \\
\hline P5 & $4,99 \mathrm{ab} \mathrm{A}$ & 4,39 a $A$ & $78,73 a b c B$ & $81,35 a b c A$ & $49,80 \mathrm{ab} B$ & $53,89 \mathrm{ab} A$ \\
\hline P6 & $4,19 a b \mathrm{~A}$ & 5,12 a $A$ & $78,80 \mathrm{abc} A$ & $80,35 \mathrm{bcA}$ & $51,09 \mathrm{ab} A$ & $53,34 \mathrm{ab} A$ \\
\hline P7 & $4,57 \mathrm{ab} A$ & 5,52 a $A$ & $79,88 \mathrm{ab} A$ & 81,73 abc $A$ & $51,10 a b B$ & 53,76 ab A \\
\hline P8 & $4,20 \mathrm{ab} A$ & 4,02 a A & $79,82 \mathrm{abc} B$ & $82,53 a b c A$ & 51,66 ab B & $55,19 \mathrm{ab} A$ \\
\hline$T$ & $4,07 \mathrm{ab} A$ & 4,34 a $A$ & 77,29 abc B & $80,23 \mathrm{bcA}$ & $48,32 \mathrm{ab} B$ & $53,22 \mathrm{ab} A$ \\
\hline
\end{tabular}

\%CIN: porcentagem de cinza; \%FDN: porcentagem de fibra em detergente neutro; \%FDA: porcentagem de fibra em detergente ácido. Médias seguidas pelas mesmas letras maiúsculas na horizontal $\mathrm{e}$ minúsculas na vertical não diferem estatisticamente entre si pelo teste Tukey ao nível de $5 \%$ de probabilidade.

estaria selecionando genótipos de desempenho mais estáveis em relação à característica \%FDN.

Em relação à \%FDA, apenas os genitores $\mathrm{P} 2$ com média de $47,38 \%$, e P3, com média de $52,58 \%$, diferiram entre si. Diferentemente do que ocorreu no $1^{\circ}$ corte, no $2^{\circ}$ corte foi verificado que os híbridos H9, H10, H14 e o genitor P2 diferiram do genitor Taiwan A-144, cuja média foi de 57,33\%. $\mathrm{Na}$ análise simultânea dos cortes assim como na \%FDN, na
$\%$ FDA, os genótipos apresentaram médias mais elevadas no $2^{\circ}$ corte, e apenas os genótipos H3, H10 e P6 não diferiram entre os cortes (Tabela 5).

$\mathrm{Na}$ característica \%CEL, em relação ao $1^{\circ}$ corte, na Tabela 6 , a média variou entre 43,19 e $38,53 \%$. Foram constatadas diferenças entre os genótipos H5, H7, H9, H14, H16, P2 e o genitor $\mathrm{P} 3$, que apresentou o maior valor médio observado. No $2^{\circ}$ corte, os híbridos H2, H7, H9, H10, H14, P2 e a testemunha

Tabela 6. Valores médios das três características da qualidade da biomassa dos 16 híbridos, dos oito genitores de capim-elefante e da testemunha (BAG-86)

\begin{tabular}{|c|c|c|c|c|c|c|}
\hline & \multicolumn{2}{|c|}{$\%$ CEL } & \multicolumn{2}{|c|}{ \%НЕМ } & \multicolumn{2}{|c|}{$\%$ LIG } \\
\hline & $\mathbf{1}^{\mathbf{0}}$ & $2^{\circ}$ & $1^{0}$ & $2^{\circ}$ & $1^{0}$ & $2^{\circ}$ \\
\hline $\mathrm{H1}$ & $39,94 a b$ B & $45,47 a b$ A & $28,29 \mathrm{ab} \mathrm{A}$ & 26,87 a B & 8,75 a $A$ & $8,50 a b A$ \\
\hline $\mathrm{H} 2$ & 39,81 ab B & $44,10 \mathrm{~b} \mathrm{~A}$ & $28,74 \mathrm{ab} A$ & 27,20 a B & 9,35 a $A$ & $7,87 \mathrm{ab} B$ \\
\hline $\mathrm{H} 3$ & 41,27 ab B & $44,58 \mathrm{ab} A$ & $27,85 \mathrm{ab} A$ & 27,65 a $A$ & 9,27 a $A$ & $8,00 \mathrm{ab} B$ \\
\hline $\mathrm{H} 4$ & 41,14 ab B & $45,51 \mathrm{ab} A$ & $28,59 \mathrm{ab} A$ & 26,43 a B & 8,96 a $A$ & $7,86 \mathrm{ab} \mathrm{A}$ \\
\hline $\mathrm{H} 5$ & 39,70 b B & $44,73 a b$ A & $29,06 \mathrm{ab} A$ & 27,03 a B & 8,63 a $A$ & $7,72 a b A$ \\
\hline $\mathrm{H} 6$ & $40,20 \mathrm{ab} B$ & $46,4 a b A$ & $28,83 \mathrm{ab} A$ & 26,90 a B & 7,97 a $A$ & 9,07 a $A$ \\
\hline $\mathrm{H} 7$ & 39,11 b B & $43,75 \mathrm{bA}$ & $27,88 \mathrm{ab} A$ & 27,19 a $A$ & 8,40 a $A$ & $7,92 \mathrm{ab} A$ \\
\hline $\mathrm{H} 8$ & $40,54 \mathrm{ab} B$ & $46,92 \mathrm{ab} A$ & $28,59 \mathrm{ab} A$ & 26,56 a B & 9,11 a $A$ & 8,96 a $A$ \\
\hline $\mathrm{H} 9$ & 39,34 b B & 43,66 b A & $28,39 \mathrm{ab} A$ & 28,21 a $A$ & 8,60 a $A$ & $7,12 \mathrm{ab} B$ \\
\hline $\mathrm{H} 10$ & $40,67 a b B$ & $43,53 \mathrm{~b} A$ & $28,26 \mathrm{ab} A$ & 27,43 a $A$ & 9,10 a $A$ & 6,24 b B \\
\hline H11 & $40,19 a b$ B & $44,98 \mathrm{ab} \mathrm{A}$ & 29,73 a $A$ & 27,77 a B & 8,58 a $A$ & $7,41 \mathrm{ab} B$ \\
\hline $\mathrm{H} 12$ & 39,79 ab B & $44,24 \mathrm{ab} A$ & $28,88 \mathrm{ab} A$ & 27,13 a $B$ & 8,44 a $A$ & $7,37 \mathrm{ab} A$ \\
\hline $\mathrm{H} 13$ & $40,35 \mathrm{ab} B$ & $45,60 \mathrm{ab} A$ & $28,28 \mathrm{ab} A$ & 26,56 a $B$ & 8,32 a $A$ & $8,35 a b A$ \\
\hline $\mathrm{H} 14$ & 39,28 b B & $44,03 \mathrm{~b} \mathrm{~A}$ & 29,60 a $A$ & 27,66 a B & 8,16 a $A$ & $6,95 a b B$ \\
\hline H15 & $40,10 a b B$ & $44,82 \mathrm{ab} A$ & $28,64 \mathrm{ab} A$ & 27,10 a $B$ & 8,69 a $A$ & $7,81 \mathrm{ab} A$ \\
\hline $\mathrm{H} 16$ & 39,42 b B & $47,06 \mathrm{ab} \mathrm{A}$ & 27,20 b B & 28,45 a $A$ & 8,82 a $A$ & $8,20 a b A$ \\
\hline P1 & $41,11 \mathrm{ab} B$ & 47,74 a A & $28,09 \mathrm{ab} A$ & 26,22 a B & 8,94 a $A$ & 8,94 a A \\
\hline P2 & 38,53 b B & 43,72 b A & $28,46 a b A$ & 27,77 a $A$ & 7,97 a $A$ & $6,69 a b B$ \\
\hline P3 & 43,19 a B & $46,63 \mathrm{ab} A$ & $29,08 \mathrm{ab} A$ & 27,68 a B & 9,06 a $A$ & $7,34 a b$ B \\
\hline P4 & $40,42 \mathrm{ab} B$ & $45,21 \mathrm{ab} A$ & $27,70 \mathrm{ab} A$ & 26,86 a $A$ & 9,27 a $A$ & $8,51 \mathrm{ab} A$ \\
\hline P5 & $40,66 \mathrm{ab} B$ & $45,61 \mathrm{ab} A$ & $28,93 \mathrm{ab} A$ & 27,45 a $B$ & 8,56 a $A$ & $7,68 a b A$ \\
\hline P6 & $40,59 \mathrm{ab} B$ & $44,60 \mathrm{ab} A$ & $27,71 \mathrm{ab} A$ & 27,01 a $A$ & 9,01 a $A$ & $7,75 a b B$ \\
\hline P7 & $40,80 \mathrm{ab} B$ & $45,54 \mathrm{ab} A$ & $28,78 \mathrm{ab} A$ & 27,97 a $A$ & 9,13 a $A$ & 7,46 ab B \\
\hline P8 & $40,88 \mathrm{ab} B$ & $46,18 \mathrm{ab} A$ & $28,16 \mathrm{ab} A$ & 27,33 a $A$ & 9,54 a $A$ & $8,37 \mathrm{ab} B$ \\
\hline$T$ & 39,32 b B & $44,13 \mathrm{bA}$ & $28,96 \mathrm{ab} A$ & 27,00 a B & 8,32 a $A$ & $8,02 \mathrm{ab} A$ \\
\hline
\end{tabular}

\%CEL: porcentagem de celulose; \%HEM: porcentagem de hemicelulose; \%LIG: porcentagem de lignina. Médias seguidas pelas mesmas letras maiúsculas na horizontal e minúsculas na vertical não diferem estatisticamente entre si pelo teste Tukey ao nível de $5 \%$ de probabilidade. 
BAG86 diferiram estatisticamente do genitor P6, cuja média foi de 47,74\%. Ao analisar os dois cortes em conjunto, observouse que todos os genótipos apresentaram maior teor de celulose na matéria seca do $2^{\circ}$ corte. A média para a $\% \mathrm{HEM}$ foi de $27,88 \%$ (Tabela 6). Nesta característica, a média variou de 29,73 a 27,20\%. Os híbridos H11 e H14, com médias de 29,73\% e $29,60 \%$, respectivamente, foram diferentes estatisticamente do híbrido H16, cuja média observada foi de $27,20 \%$. No $2^{\circ}$ corte, a média variou de $28,45 \%$ a $26,22 \%$. Os genótipos apresentaram valores médios mais baixos se comparados ao $1^{\circ}$ corte. Além disso, não foram detectadas diferenças entre os genótipos estudados. Já na análise dos dois cortes, para esta característica, os resultados foram melhores no $1^{\circ}$ corte, e alguns genótipos como H3, H7, H9, H10, P2, P4, P6, P5 e P8 não sofreram influência do corte, ou seja, os valores médios dos dois cortes foram iguais estatisticamente. Além dessa estabilidade para a característica \%CEL, segundo Oliveira et al. (2014) os genótipos Porto Rico 534-B e Taiwan A-146 apresentam alta produção de matéria seca anual, podendo ser classificado como sendo do grupo elite para a produção de biomassa energética.

Para a característica \%LIG, na comparação das médias, os genótipos não diferiram no $1^{\circ}$ corte, enquanto no $2^{\circ}$ corte, foram observadas algumas diferenças e, neste caso, os híbridos H6, H8 e o genitor P6 diferiram do híbrido H10, cuja média foi de 6,24\%. Daher et al. (2014) ao avaliarem a capacidade de combinação em capim-elefante por meio da análise dialélica constataram que a combinação hibrida resultante ente Vruckwona x Taiwan A-146, H6 no presente estudo, apresenta alto teor de fibras e que o genótipo P6 pode ser utilizado em programas de melhoramento cujo objetivo seja a melhoria da qualidade da biomassa em capim-elefante para fins energéticos. Ao analisar o comportamento dos genótipos nos dois cortes simultaneamente, observou-se que eles apresentaram maior teor de lignina na matéria seca do $1^{\circ}$ corte. Nos híbridos $\mathrm{H} 1$, H4, H5, H6, H7, H8, H12, H13, H15, H16 e nos genitores P6, P4, P5 e a testemunha BAG-86, as médias do teor de lignina não diferiram entre os cortes, sendo considerados estáveis em relação a essa característica (Tabela 6).

\section{Conclusões}

As combinações híbridas H1, H3, H5, H7, H9 e os genitores P4 e P6 possuem qualidades satisfatórias de biomassa e, com isso, apresentam potencial para fins energéticos.

\section{Literatura Citada}

Assis, L. C. S. L. C.; Lira, M. A.; Santos, M. V. F.; Dubeux Júnior, J. C. B.; Cunha, M. V. Estimativa de parâmetros genéticos sob duas estratégias de avaliação em híbridos intra e interespecíficos de capim-elefante. Revista Brasileira de Zootecnia, v.39, n.12, p.2589-2597, 2010. <http://dx.doi. org/10.1590/S1516-35982010001200005>.

Cardona, E. M.; Rios, L. A.; Peña, J. D. Disponibilidad de variedades de pastos y forrajes como potenciales materiales lignocelulósicos para la producción de bioetanol en Colombia. Información Tecnológica, v.23, n.6, p.87-96, 2012. <http:// dx.doi.org/10.4067/S0718-07642012000600010>.
Cruz C. D. GENES - a software package for analysis in experimental statistics and quantitative genetics. Acta Scientiarum. Agronomy, v.35, n.3, p.271-276, 2013. $<$ http://dx.doi.org/10.4025/actasciagron.v35i3.21251>.

Daher, R. F.; Souza, L. B.; Gravina, G. A.; Machado, J. C.; Ramos, H. C. C.; Silva, V. Q. R.; Menezes, B. R. S.; Schneider, L. S. A.; Oliveira, M. L. F.; Gottardo, R. D. Use of elephant grass for energy production in Campos dos Goytacazes-RJ, Brazil. Genetics and Molecular Research, v.13, n.4, p.10898-10908, 2014. <http://dx.doi. org/10.4238/2014>.

Flores, R. A.; Urquiaga, S.; Alves, B. J. R.; Collier, L. S.; Zanetti, J. B.; Prado, Mello, R. Nitrogênio e idade de corte na qualidade da biomassa de capimelefante para fins agroenergéticos cultivado em Latossolo. Semina: Ciências Agrárias, v.34, n.1, p.127-136, 2013. <http://dx.doi. org/10.5433/1679-0359.2013v34n1p127>.

Jank, L.; Lima, E. A.; Simeão, R. M.; Andrade, R. C. Potential of Panicum maximum as a source of energy. Tropical Grasslands - Forrajes Tropicales, v.1, n.1, p.92-94, 2013. $<$ http://dx.doi.org/10.17138/TGFT(1)92-94>.

Knoll, J. E.; Anderson, W. F.; Strickland, T. C.; Hubbard, R. K.; Malik, R. Low-Input Production of Biomass from Perennial Grasses in the Coastal Plain of Georgia, USA. Bioenergy Research, v.5, n.1, p.206-214, 2012. <http:// dx.doi.org/10.1007/s12155-011-9122-x>.

Leão, F. F.; Cancellier, L. L.; Pereira, A. V.; Ledo, F. J. S.;Afférri, F. S. Produção forrageira e composição bromatológica de combinações genômicas de capim-elefante e milheto. Revista Ciência Agronômica, v.43, n.2, p.368-375, 2012. $<$ http://dx.doi.org/10.1590/S1806-66902012000200021>.

Meinerz, G. R.; Olivo, C. J.; Agnolin, C. A.; Dullius, A. P.; Moraes, R. S.; Mombach, G.; Foletto, V.; Machado, P. R. Produção e valor nutritivo da forragem de capim-elefante em dois sistemas de produção. Revista Brasileira de Zootecnia, v.40, n.12, p.2673-2680, 2011. <http://dx.doi. org/10.1590/S1516-35982011001200009>.

Menezes, B. R. S.; Daher, R. F.; Gravina, G. A.; Gottardo, R. D.; Schneider, L. S. A.; Rocha, A. S. Comportamento Per se de híbridos de capim-elefante para fins energéticos. Comunicata Scientiae, v.7, n.1, p.73-85, 2016. <http:// dx.doi.org/10.14295/CS.v7i1.946>.

Morais, R. F.; Zanetti, J. B.; Pacheco, B. M.; Jantália, C. P.; Rodrigues, B. J.; Urquiaga, S. Produção e qualidade da biomassa de diferentes genótipos de capim-elefante cultivados para uso energético. Revista Brasileira de Agroecologia, v.4, n.2, p.1103- 1107, 2009. <http:// www.aba-agroecologia.org.br/revistas/index.php/ rbagroecologia/article/viewFile/8143/5794>. 28 Jul. 2015.

Oliveira, É. S.; Rogério Figueiredo Daher; Ponciano, N. J.; Gravina, G. A.; Sant'ana, J. A. A.; Gottardo, R. D.; Menezes, B. R. S.; Souza, P. M.; Souza, C. L. M.; Silva, V. B.; Rocha, A. S.; Novo, A. A. C. Variation of morphoagronomic and biomass quality traits in elephant grass for energy purposes according to nitrogen levels. American Journal of Plant Sciences, v.6, n.11, p.1685-1696, 2015. $<$ http://dx.doi.org/10.4236/ajps.2015.611168>. 
Oliveira, M. L. F.; Daher, R. F.; Gravina, G. A.; Silva, V. B.; Viana, A. P.; Rodrigues, E. V.; Shimoya, A.; Amaral Júnior, A. T.; Menezes, B. R. S.; Rocha, A. S. Pre-breeding of elephant grass for energy purposes and biomass analysis in Campos dos Goytacazes- RJ, Brazil. African Journal of Agricultural Research, v.9, n.36, p.2743-2758, 2014. $<$ http://dx.doi.org/10.5897/AJAR2014.8900>.

Paterlini, E. M.; Arantes, M. D. C.; Gonçalves, F. G.; Vidaurre, G. B.; Bauer, M. O.; Moulin, J. C. Evaluation of elephant grass for energy use. Journal of Biotechnology and Biodiversity, v.4, n .2: p.119-125, 2013. <http://revista.uft. edu.br/index.php/JBB/article/viewFile/401/325>. 28 Jul. 2015.

Pereira, A. V.; Valle, C. B.; Ferreira, R. P.; Miles, J. W. Melhoramento de forrageiras tropicais. In: Nass, L. L.; Valois, A. C. C.; Melo, I. S.; Valadares Ingres, M. C. (Eds.). Recursos Genéticos e Melhoramento de Plantas. Rondonópolis: Fundação Mato Grosso, 2001, p.549-602.

Pimentel-Gomes, F. Curso de estatística experimental. Piracicaba: FEALQ, 2009. 451p.
Rossi, D. A.; Menezes, B. R. S.; Daher, R. F.; Gravina, G. A.; Lima, R. S. N.; Ledo, F. J. S.; Gottardo, R. D.; Campostrini, E.; Souza, C. L. M. Canonical correlations in elephant grass for energy purposes. African Journal of Biotechnology, v.13, n.36, p.36663671, 2014. <http://dx.doi.org/ 10.5897/AJB2014.13915>.

Silva, A. L. C.; Santos, M. V. F.; Dubeux Júnior, J. C. B.; Lira, M. A.; Ferreira, R. L. C.; Freitas, E. V.; Cunha, M. V.; Silva, M. C. Variabilidade e herdabilidade de caracteres morfológicos em clones de capim-elefante na Zona da Mata de Pernambuco. Revista Brasileira de Zootecnia, v.39, n.1, p.2132-2140, 2010. <http://dx.doi.org/10.1590/S1516-35982010001000005>.

Silva, D. J.; Queiroz, A. C. Análise de alimentos: métodos químicos e biológicos. 3. ed. Viçosa, 2002. 235p.

Steel, R. G. D; Torrie, J.H. Principles and procedures of statistics. 2.ed. New York: McGraw-Hill, 1980.633p.

Urquiaga S.; Alves, B.; Boddey R.M. Capim elefante: uma nova fonte alternativa de energia. Ambiente Brasil. $<$ http:// ambientes.ambientebrasil.com.br/agropecuario/artigo_ agropecuario/capim_elefante\%3A_uma_nova_fonte_ alternativa_de_energia.html $>.23$ Jul. 2015. 